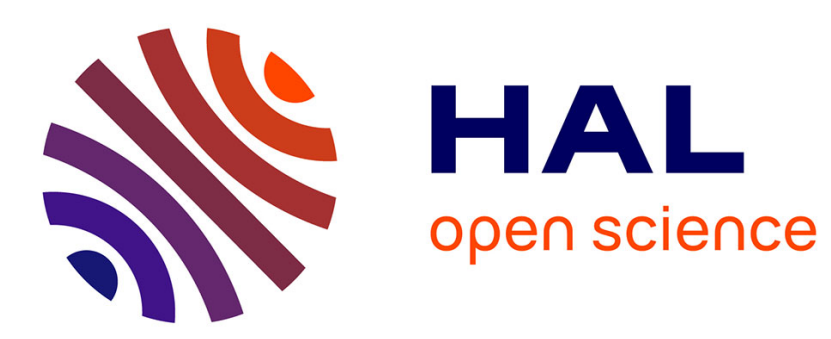

\title{
Universal formula for robust stabilization of affine nonlinear multistable systems
}

Nelson F Barroso, Rosane Ushirobira, Denis Efimov

\section{To cite this version:}

Nelson F Barroso, Rosane Ushirobira, Denis Efimov. Universal formula for robust stabilization of affine nonlinear multistable systems. Conference on Decision and Control, Dec 2019, Nice, France. hal-02340333

\section{HAL Id: hal-02340333 \\ https://hal.inria.fr/hal-02340333}

Submitted on 30 Oct 2019

HAL is a multi-disciplinary open access archive for the deposit and dissemination of scientific research documents, whether they are published or not. The documents may come from teaching and research institutions in France or abroad, or from public or private research centers.
L'archive ouverte pluridisciplinaire HAL, est destinée au dépôt et à la diffusion de documents scientifiques de niveau recherche, publiés ou non, émanant des établissements d'enseignement et de recherche français ou étrangers, des laboratoires publics ou privés. 


\title{
Universal formula for robust stabilization of affine nonlinear multistable systems
}

\author{
Nelson F. Barroso, Rosane Ushirobira, Denis Efimov
}

\begin{abstract}
In this paper, the problem of robust stabilization of affine nonlinear multistable systems with respect to disturbance inputs is studied. The results are obtained using the framework of input-to-state stability (ISS) and integral input-to-state stability (iISS) for systems with multiple invariant sets. The notions of ISS and iISS control Lyapunov functions as well as the small control property are extended within the multistability framework. It is verified that the universal control formula can be applied to yield the ISS (iISS) property to the closedloop system. The efficiency of the proposed control Lyapunov function in the multistable sense is illustrated in two academic examples.
\end{abstract}

\section{INTRODUCTION}

Due to its importance for several scientific disciplines ranging from mechanics and electronics [19], [13] to biology [26], [20], [24] and neurosciences [25], the analysis of stability and robustness properties of multistable systems have become increasingly attractive from the perspective of systems and control theory. Multistable systems include bistable systems (with at least two stable equilibria) [34], [7], almost globally stable systems (with only one attracting invariant set) [2], and nonlinear systems with generic invariant sets [4], [9], [14], [17], [18], [27], [32], [33]. In [12], it was proposed a global asymptotic stability notion as well as the necessary and sufficient Lyapunov characterization for multistable systems, having as the object of investigation all compact invariant solutions of the system (including locally stable and unstable ones).

By virtue of nontrivial relationships between different regions that compose its state space, and the complex intertwined boundaries between then, multistable systems are extremely sensitive to initial conditions and perturbations. The characterization introduced in [12] has been also proved to be useful in robustness analysis with respect to external disturbances. In fact, it was made clear in [3] that the most natural way to solve this problem is to relax the Lyapunov stability requirement on relatively mild additional assumptions on the decomposition of invariant sets. This intuitive path has led to a new line of research which starts from the characterizations of input-to-state stability (ISS) for this class of systems in terms of usual Lyapunov dissipation

N. F. Barroso, R. Ushirobira and D. Efimov are with Inria, University of Lille, CNRS, UMR 9189 - CRIStAL, F-59000 Lille, France. D. Efimov is also with ITMO University, 197101 Saint Petersburg, Russia. This work was supported by the ANR project WaQMoS (ANR 15 CE 04 0002). It was also supported by the Ministry of Science and Higher Education of Russian Federation, passport of goszadanie no. 2019-0898. N. F. Barroso is partly supported by the regional council of Hauts-de-France. inequalities, generalizing the classical ISS theory [30], [31], [8], [3], [15]. In its turn, integral input-to-state stability (iISS) characterization, which is weaker than the classical one given in [29], [21], [5], was extended in [16] for systems with multiple invariant sets.

Once ISS and iISS characterizations in the multistable sense are already available, the research interest on the problem of designing robust stabilizing control laws in this framework naturally increases. In this setting, such a problem consists in finding state feedback control laws that make the closed loop system ISS or iISS stable with respect to a family of finite disjoint compact invariant sets against external disturbances effects. In the classical approach, most of this activity is centered around the control Lyapunov function (CLF) theory [6], [28], [30], [11], [10]. In [6], it was shown that the existence of a CLF leads to an explicit formulation for stabilizing control laws. Similar results were proven in [22] for the ISS (iISS) case resulting in appropriated universal formulation for the assignment of a ISS (iISS) CLF rendering ISS (iISS) properties to the closedloop system. In the present work, we are interested in the robust stabilization of multistable affine nonlinear systems with respect to disturbance inputs. Our approach is based on the theory developed in [12], [3], [16] and it aims to find conditions of CLF existence in the context of systems with multiple invariant sets (compact and maybe disconnected) and show how such a control can be explicitly designed for robust stabilization in ISS (iISS) sense.

The outline of this work is as follows. The main definitions and problem statement are given in Section [II while the obtained results are presented in Section [III In section IV are presented two examples of application of the proposed CLF approach. Final remarks and discussion are summarized in Section $\mathrm{V}$

\section{Definitions and Problem Statement}

Let $\mathcal{M}$ be a $n$-dimensional smooth manifold without boundary, equipped with a metric $\delta: \mathcal{M} \times \mathcal{M} \rightarrow \mathbb{R}_{+}$, $\mathbb{R}_{+}=\{s \in \mathbb{R}: s \geq 0\}$. Consider a nonlinear model of dynamical systems evolving on this manifold:

$$
\begin{aligned}
\dot{x}(t) & =f(x(t), u(t)), \quad \forall t \in \mathbb{R}_{+}, \\
y(t) & =h(x(t)),
\end{aligned}
$$

where $x(t) \in \mathcal{M}$ is the state vector, $u(t) \in \mathbf{U} \subseteq \mathbb{R}^{m}$ is the input vector, $u$ is an element of $\mathcal{U}$, the set of admissible controls $\mathbb{R}_{+} \rightarrow \mathbf{U}$ (locally essentially bounded and measurable signals), and $y(t) \in \mathcal{Y} \subseteq \mathbb{R}^{p}$ is the output vector. Let 
$f: \mathcal{M} \times \mathbf{U} \rightarrow T_{x} \mathcal{M}$ be a locally Lipschitz continuous function on $\mathcal{M}$ (here $T_{x} \mathcal{M}$ denotes the tangent space of $\mathcal{M}$ at $x$ ), and assume that $h: \mathcal{M} \rightarrow \mathcal{Y}$ is continuously differentiable, $h(0)=0$ and $f(0,0)=0$.

Denote by $x\left(t, x_{0} ; u\right)$ the uniquely defined solution of (1) at time $t \geq 0$ such that $x(0)=x_{0}$ under the input $u \in \mathcal{U}$. For the unperturbed system, i.e. the system (1) with $u \equiv 0$, we have:

$$
\dot{x}(t)=f(x(t), 0), \quad t \geq 0,
$$

and we say that $S \subset \mathcal{M}$ is invariant if for all $x_{0} \in S$, $x\left(t, x_{0} ; 0\right) \in S$ for all $t \in \mathbb{R}$.

For a set $S \subset \mathcal{M}$ and $x \in \mathcal{M}$ define the corresponding distance as

$$
|x|_{S}=\inf _{a \in S} \delta(x, a) .
$$

For a measurable function $g: \mathbb{R}_{+} \rightarrow \mathbb{R}^{m}$, define its $L_{\infty^{-}}$ norm as

$$
\|g\|_{\infty}=\operatorname{ess} \sup _{t \geq 0}|g(t)|
$$

\section{A. Decomposition of a compact invariant set}

Let $\Lambda \subset \mathcal{M}$ be a compact invariant set for the unperturbed system (3). To characterize the evolution of this system along $\mathcal{M}$, it is useful to decompose $\Lambda$ and explicitly determine the existence of solutions traveling between different components of its decomposition.

Definition 1. [23] A decomposition of $\Lambda$ is a finite, disjoint family of compact invariant sets $\Lambda_{1}, \cdots, \Lambda_{k}$ such that $\Lambda=$ $\bigcup_{i=1}^{k} \Lambda_{i}$.

For an invariant set $\Lambda$, its attracting and repulsing subsets can be defined, respectively, as follows:

$$
\begin{aligned}
& \mathfrak{A}(\Lambda)=\left\{x_{0} \in \mathcal{M}:\left|x\left(t, x_{0} ; 0\right)\right|_{\Lambda} \rightarrow 0 \text { as } t \rightarrow+\infty\right\}, \\
& \mathfrak{R}(\Lambda)=\left\{x_{0} \in \mathcal{M}:\left|x\left(t, x_{0} ; 0\right)\right|_{\Lambda} \rightarrow 0 \text { as } t \rightarrow-\infty\right\} .
\end{aligned}
$$

Based on these definitions, we can define a relation between two invariant sets $\mathcal{W} \subset \mathcal{M}$ and $\mathcal{D} \subset \mathcal{M}$ by $\mathcal{W} \prec \mathcal{D}$ if $\mathfrak{A}(\mathcal{W}) \cap \mathfrak{R}(\mathcal{D}) \neq \emptyset$. This relation implies that there is a solution connecting the set $\mathcal{D}$ with the set $\mathcal{W}$. A collection of $r$ disjoint sets that can be reached from one to another in a loop by a suitable concatenation of systems solutions is called $r$-cycle.

Definition 2. [23] Let $\Lambda_{1}, \cdots, \Lambda_{k}$ be a decomposition of $\Lambda$.

1) An $r$-cycle $(r \geq 2)$ is an ordered $r$-tuple of distinct indices $i_{1}, \cdots, i_{r}$ such that $\Lambda_{i_{1}} \prec \cdots \prec \Lambda_{i_{r}} \prec \Lambda_{i_{1}}$.

2) A 1-cycle is an index $i$ such that $\left(\mathfrak{R}\left(\Lambda_{i}\right) \cap \mathfrak{A}\left(\Lambda_{i}\right)\right) \backslash$ $\Lambda_{i} \neq \emptyset$.

3) A filtration ordering is a numbering of the $\Lambda_{i}$ so that $\Lambda_{i} \prec \Lambda_{j} \Rightarrow i \leq j$.

However, what qualifies a decomposition for its treatment by means of Lyapunov-like analytical tools is the absence of these cycles. Therefore, we will next consider the following assumption:
Assumption 1. [3] A compact invariant set $\mathcal{W}$ containing all $\alpha$-and $\omega$-limit sets of the unperturbed system (3), admits a finite decomposition without cycles: $\mathcal{W}=\bigcup_{i=1}^{k} \mathcal{W}_{i}$ for some non-empty disjoint compact sets $\mathcal{W}_{i}$ which forms a filtration ordering of $\mathcal{W}$, as detailed in Definition 2 .

\section{B. Robust stability notions for a decomposable compact invariant set $\mathcal{W}$}

In this subsection, we list several ISS and iISS stability properties for (1) with respect to $\mathcal{W}$ satisfying Assumption 1 Most of these properties are direct extensions of the classical ISS and iISS notions introduced in [30], [31], [21], [5]. The definitions of function classes $\mathcal{K}$ and $\mathcal{K}_{\infty}$ can be found in [8]. A function $V: \mathcal{M} \rightarrow \mathbb{R}_{+}$is called positive definite if it vanishes only at the origin, and proper unbounded if $V(x) \rightarrow+\infty$ for $|x|_{\mathcal{W}} \rightarrow+\infty$. The Lie derivative of a continuously differentiable function $V$ along a vector field $f: \mathcal{M} \rightarrow \mathbb{R}^{n}$ is denoted by:

$$
D V(x) f(x, u)=\frac{\partial V(x)}{\partial x} f(x, u) .
$$

Definition 3. [3], [16] The system (1) has the practical asymptotic gain $(p A G)$ property if there exist $\eta \in \mathcal{K}_{\infty}$ and $q \geq 0$ such that for all $x_{0} \in \mathcal{M}$ and all $u \in \mathcal{U}$, the solutions are defined for all $t \geq 0$ and the following holds:

$$
\limsup _{t \rightarrow+\infty}\left|x\left(t, x_{0} ; u\right)\right|_{\mathcal{W}} \leq \eta\left(\|u\|_{\infty}\right)+q
$$

If $q=0$, then we say that the asymptotic gain ( $A G)$ property holds. Moreover, if (4) is satisfied for $q=0$ for the system (3) only, then we will say that (1) has the zero-global attraction $(0-G A T T)$ property with respect to a compact invariant set $\mathcal{W}$.

Definition 4. [3] The system (1) has the limit property (LIM) with respect to $\mathcal{W}$ if there exists $\mu \in \mathcal{K}_{\infty}$ such that for all $x_{0} \in \mathcal{M}$ and all $u \in \mathcal{U}$ the solutions are defined for all $t \geq 0$ and the following holds:

$$
\inf _{t \geq 0}\left|x\left(t, x_{0} ; u\right)\right|_{\mathcal{W}} \leq \mu\left(\|u\|_{\infty}\right) .
$$

Definition 5. [3] The system (1) has the practical global stability $(p G S)$ property with respect to $\mathcal{W}$ if there exists $\beta \in \mathcal{K}_{\infty}$ and $c \geq 0$ such that for all $x_{0} \in \mathcal{M}$ and all $u \in \mathcal{U}$, the following holds for all $t \geq 0$ :

$$
\left|x\left(t, x_{0} ; u\right)\right|_{\mathcal{W}} \leq \beta\left(\max \left\{\left|x_{0}\right|_{\mathcal{W}}+c, \|\left. u\right|_{\infty}\right\}\right) .
$$

Definition 6. [3], [16] A $\mathcal{C}^{1}$ function $V: \mathcal{M} \rightarrow \mathbb{R}$ is a practical ISS Lyapunov function for (1) if there exist $\mathcal{K}_{\infty}$ functions $\alpha_{1}, \alpha_{2}, \alpha_{3}$ and $\gamma$, and $q \geq 0, c \geq 0$ such that

$$
\alpha_{1}\left(|x|_{\mathcal{W}}\right) \leq V(x) \leq \alpha_{2}\left(|x|_{\mathcal{W}}\right)+c
$$

and the following dissipation inequality holds for all $(x, u) \in$ $\mathcal{M} \times \mathcal{U}$ :

$$
D V(x) f(x, u) \leq-\alpha_{3}\left(|x|_{\mathcal{W}}\right)+\gamma(|u|)+q .
$$


If (6) holds for $q=0$, then $V$ is said to be an ISS Lyapunov function. If (6) holds for $q=0$ and a positive definite function $\alpha_{3}: \mathbb{R}_{+} \rightarrow \mathbb{R}_{+}$, then $V$ is said to be an iISS Lyapunov function.

Definition 7. [16] The system (11) has the uniform bounded energy bounded-state (UBEBS) property if for some $\alpha, \gamma, \sigma \in$ $\mathcal{K}_{\infty}$ and some positive constant $c$, the following estimate holds for all $t \geq 0$, all $x_{0} \in \mathcal{M}$ and all $u \in \mathcal{U}$ :

$$
\alpha\left(\left|x\left(t, x_{0} ; u\right)\right|_{\mathcal{W}}\right) \leq \gamma\left(\left|x_{0}\right| \mathcal{W}\right)+\int_{0}^{t} \sigma(|u(\tau)|) d \tau+c .
$$

Definition 8. [16] The system (10, (2) has the smooth dissipativity property if there exist a $\mathcal{C}^{1}$ function $V: \mathcal{M} \rightarrow \mathbb{R}_{+}$, $\alpha_{1}, \alpha_{2}, \sigma \in \mathcal{K}_{\infty}$, a continuous positive definite function $\alpha_{4}$, and a continuous output map $h: \mathcal{M} \rightarrow \mathbb{R}^{p}$ with

$$
|x|_{\mathcal{W}}=0 \Rightarrow h(x)=0, \quad \forall x \in \mathcal{M}
$$

such that (5) is satisfied for all $x \in \mathcal{M}$ and the following dissipation inequality holds for all $(x, u) \in \mathcal{M} \times \mathcal{U}$ :

$$
D V(x) f(x, u) \leq-\alpha_{4}(|h(x)|)+\sigma(|u|) .
$$

Definition 9. [16] The system (1), (2) has the weak zerodetectability property if the following relation holds:

$$
h\left(x\left(t, x_{0} ; 0\right)\right) \equiv 0, \forall t \geq 0 \Rightarrow\left|x\left(t, x_{0} ; 0\right)\right|_{\mathcal{W}} \rightarrow 0
$$

as $t \rightarrow \infty$.

The principal results connecting these properties are as following:

Theorem 1. [3] Consider a nonlinear system (17) and let $\mathcal{W}$ be as in Assumption 1 Then the following are equivalent:

1) The system enjoys the $p A G$ or $A G$ property.

2) The system admits an ISS Lyapunov function.

3) The system admits an ISS Lyapunov function constant on invariant sets.

4) The system admits a practical ISS Lyapunov function.

5) The system enjoys the LIM property and the $p G S$.

The system as in (1) that satisfies these properties will be called ISS in the multistable sense with respect to the set $\mathcal{W}$ and input $u$.

Theorem 2. [16] Consider a nonlinear system (1) and let $\mathcal{W}$ be as in Assumption $\square$ Then the following facts are equivalent:

1) 0-GATT and UBEBS properties.

2) Existence of an iISS Lyapunov function $V$ such that $D V(x)=0$ for all $x \in \mathcal{W}$.

3) Existence of an iISS Lyapunov function $V$.

4) Existence of an output function that makes the system smoothly dissipative and weakly zero-detectable.

The system as in (1) that satisfies these properties will be called iISS in the multistable sense with respect to the set $\mathcal{W}$ and the input $u$.

\section{Problem statement}

In this paper, we deal with a subclass of nonlinear dynamical systems (1), (2) affine in the input of the following form:

$$
\begin{aligned}
& \dot{x}(t)=f(x(t), v(t))+G(x(t)) u(t), \quad t \in \mathbb{R}_{+}, \\
& y(t)=h(x(t)),
\end{aligned}
$$

where $x(t) \in \mathcal{M}$ is the state vector, $v(t) \in \mathbb{R}^{k}$ is a disturbance, $u(t) \in \mathbf{U} \subseteq \mathbb{R}^{m}$ is the input vector, $u$ is an element of $\mathcal{U}$, the set of admissible controls $\mathbb{R}_{+} \rightarrow \mathbf{U}$ (locally essentially bounded and measurable signals), and $y(t) \in \mathcal{Y} \subseteq \mathbb{R}^{p}$ is the output vector. For this system $f: \mathcal{M} \times \mathbb{R}^{k} \rightarrow \mathbb{R}^{n}$ and the columns of the matrix function $G: \mathcal{M} \rightarrow \mathbb{R}^{n \times m}$ are assumed to be locally Lipschitz continuous on $\mathcal{M}, h: \mathcal{M} \rightarrow \mathbb{R}^{p}$ is continuously differentiable, $h(0)=0$, and $f(0,0)=0$.

Systems in the form (8), (9) are said to be ISS (iISS) stabilizable if there exists a control law $u=K(x)$, with $K: \mathcal{M} \rightarrow \mathbf{U} \subseteq \mathbb{R}^{m}$, so that the closed-loop system has the ISS (iISS) property in $v \in \mathbb{R}^{k}$ with respect to $\mathcal{W}$. Therefore, the problem studied in this work can be formally determined as follows:

Problem. Consider the systems described by the affine nonlinear model (8), (9). Under Assumption 1, find conditions for these systems to be ISS (iISS) stabilizable.

\section{MAIN RESULTS}

As for ISS (iISS) stabilization with respect to a compact set [21], [28], we will look for existence conditions of a stabilizing feedback using the CLF framework. To present our main result, we introduce a suitable notion of ISS (iISS) CLF from the point of view of multistability framework.

Definition 10. A practical ISS CLF for the system (8), (9) and control $u(t) \in \mathbf{U} \subseteq \mathbb{R}^{m}$ is a differentiable function $V: \mathcal{M} \rightarrow \mathbb{R}_{+}$satisfying:

1) There exist class $\mathcal{K}_{\infty}$ functions $\alpha_{1}$ and $\alpha_{2}$ such that (5) holds for all $x \in \mathcal{M}$.

2) There exist class $\mathcal{K}_{\infty}$ functions $\chi$ and $\alpha_{3}$ and a constant $q \geq 0$ such that for all $x \in \mathcal{M}$ and all $v \in \mathbb{R}^{k}$

$$
\begin{aligned}
& \inf _{u \in \mathcal{U}}\{a(x, v)+b(x) u\} \leq-\alpha_{3}(\mid\left.\left.x\right|_{\mathcal{W}}\right) \\
&+\chi(|v|)+q,
\end{aligned}
$$

where $a(x, v)=D V(x) f(x, v), b(x)=D V(x) G(x)$. If 10 holds for $q=0$, then $V$ is an ISS CLF. In addition, such $V$ is an iISS CLF if the both items are satisfied for $q=0$ and a continuous positive definite function $\alpha_{3}$.

The inequality (10) can be rewritten as follows:

$$
\inf _{u \in \mathcal{U}}\{a(x, v)-\tilde{\chi}(|v|)-q+b(x) u\} \leq-\alpha_{3}\left(|x|_{\mathcal{W}}\right),
$$

where $\tilde{\chi} \in \mathcal{K}_{\infty}$ is such that $\tilde{\chi}(s) \geq \chi(s)$ for all $s \in \mathbb{R}_{+}$and there exists a function

$$
\psi(x)=\sup _{v \in \mathbb{R}^{k}}\{a(x, v)-\tilde{\chi}(|v|)-q\},
$$


e.g., take $\tilde{\chi}(s)=\max \left\{\chi(s), \sup _{|x|,|v| \leq s}|a(x, v)|\right\}[21]$, then with such a choice $\psi(x)=\sup _{|v| \leq|x|}\{a(x, v)-\tilde{\chi}(|v|)-q\}$. By construction $\psi(x) \leq 0$ for all $x \in \mathcal{W}$, and since the function $a$ is at least Lipschitz continuous in $x$, then the function $\psi$ admits the same property. In addition, for all $x \in$ W

$$
\inf _{u \in \mathcal{U}}\{\psi(x)+b(x) u\} \leq-\alpha_{3}\left(|x|_{\mathcal{W}}\right) .
$$

Finally, define

$$
\Psi(x)=\psi(x)+\frac{1}{2} \alpha_{3}\left(|x|_{\mathcal{W}}\right)
$$

then for all $x \in \mathcal{M}$ and $v \in \mathbb{R}^{k}$ :

$$
\begin{gathered}
\Psi(x) \geq \frac{1}{2} \alpha_{3}\left(|x|_{\mathcal{W}}\right)+a(x, v)-\tilde{\chi}(|v|)-q, \\
\inf _{u \in \mathcal{U}}\{\Psi(x)+b(x) u\} \leq-\frac{1}{2} \alpha_{3}\left(|x|_{\mathcal{W}}\right) .
\end{gathered}
$$

Definition 11. A differentiable function $V: \mathcal{M} \rightarrow \mathbb{R}$ possesses a variant of the classical small control property $(S C P)$ with respect to $x \in \mathcal{M}$ if it takes constant values on any $\mathcal{W}_{i}$ with $i=1, \ldots, k$, from the decomposition of $\mathcal{W}$, and for each $\varepsilon>0$ there is a $\delta>0$ such that whenever $0<|x|_{\mathcal{W}}<\delta$ there exists some control $u \in \mathbf{U} \subseteq \mathbb{R}^{m}$ with $|u|<\varepsilon$ such that

$$
\Psi(x)+b(x) u<0 .
$$

Note that for the both above cases (ISS/iISS), the condition (10) can be replaced with another one:

$$
b(x) \equiv 0 \Rightarrow \Psi(x) \leq-\frac{1}{2} \alpha_{3}\left(|x|_{\mathcal{W}}\right),
$$

for all $x \in \mathcal{M}$, and $\alpha_{3}$ is a class $\mathcal{K}_{\infty}$ function or a positive definite function for the cases of ISS or iISS, respectively. Therefore, the condition (12) formulates the main restriction to check for ISS (iISS) CLF in the system (8), (9).

Following the CLF framework from [6], [28], we will use the "universal" control formula, which in our case takes the form:

$$
u=K(x)=\kappa\left(\Psi(x),|b(x)|^{2}\right) b(x)^{T},
$$

where

$$
\kappa(s, r)=\left\{\begin{array}{ll}
-\frac{s+\sqrt{s^{2}+r^{2}}}{r} & \text { if } x \notin \mathcal{W} \\
0 & \text { if } x \in \mathcal{W}
\end{array} .\right.
$$

Then, according with the above developments we propose the following theorem and lemma. We omit the proofs due to space limitations.

Theorem 3. Let an affine system (8) admit Assumption 1 . If the function $V: \mathcal{M} \rightarrow \mathbb{R}_{+}$is an ISS (iISS) CLF, then the feedback law (13) is continuous in $\mathcal{M} \backslash \mathcal{W}$ and it provides the ISS (iISS) property in $v$ with respect to $\mathcal{W}$ for (8). If such a CLF satisfies the SCP given in Definition 11 then the control (13) is continuous on $\mathcal{M}$.

Note that $V(x)=0$ for $x \in \mathcal{A}$ (it reaches its minimum), when $\mathcal{A} \subset \mathcal{W}$, then the control (13) will provide almost global or local attraction of the set $\mathcal{A}$ in the case $v=0$.
Our further directions of research will apply the smooth dissipativity property for iISS stabilization of (8), (9), but here we will formulate the following consequence of our main result:

Lemma 1. Consider an affine system (8) that satisfies Assumption 1] Then there exists a continuous control that provides the ISS (iISS) property in $v$ with respect to $\mathcal{W}$ for (8) if and only if the system admits an ISS (iISS) CLF with SCP.

\section{EXAMPLES}

In this section we will illustrate peculiarities and features of the proposed CLF in the multistable framework on two academic examples.

\section{A. Duffing oscillator}

Consider the following variant of Duffing oscillator:

$$
\begin{aligned}
& \dot{x}_{1}(t)=x_{2}(t)+u(t)+v_{1}(t), \\
& \dot{x}_{2}(t)=-x_{2}(t)+x_{1}(t)\left[x_{1}^{2}(t)-1\right]+v_{2}(t),
\end{aligned}
$$

where $x(t)=\left(x_{1}(t) x_{2}(t)\right)^{\top} \in \mathcal{M}=\mathbb{R}^{2}, u(t) \in \mathbb{R}$ and $v(t)=\left(v_{1}(t) v_{2}(t)\right)^{\top} \in \mathbb{R}^{2}$ have the same sense as before. It is straightforward to check that for $u=0$ and $v=0$ the system has three equilibria, thus, $\mathcal{W}=\{(-1,0),(0,0),(1,0)\}$. Linearization shows that the origin is a locally asymptotically stable, and the two equilibria, $(-1,0)$ and $(1,0)$ are unstable foci (see Fig. 1).

Select $\mathcal{A}=\{(-1,0),(1,0)\}$ as the set that will be almost globally attractive in the closed-loop system. To this end, as a CLF candidate, choose

$$
V(x)=\frac{1}{4}\left(x_{1}^{2}-1\right)^{2}+\frac{1}{2} x_{2}^{2},
$$

whose derivative for the oscillator takes the usual form $\dot{V}=$ $a(x, v)+b(x) u$, where

$$
\begin{gathered}
a(x, v)=2 x_{1}\left(x_{1}^{2}-1\right) x_{2}-x_{2}^{2}+x_{1}\left(x_{1}^{2}-1\right) v_{1}+x_{2} v_{2}, \\
b(x)=x_{1}\left(x_{1}^{2}-1\right) .
\end{gathered}
$$

Select $\chi(s)=s^{2}$, then

$$
\begin{gathered}
a(x, v)-\chi(|v|)=\left(\begin{array}{c}
x_{1}\left(x_{1}^{2}-1\right) \\
x_{2} \\
v_{1} \\
v_{2}
\end{array}\right) Q\left(\begin{array}{c}
x_{1}\left(x_{1}^{2}-1\right) \\
x_{2} \\
v_{1} \\
v_{2}
\end{array}\right) \\
-\frac{1}{4} x_{2}^{2}+2.3 x_{1}^{2}\left(x_{1}^{2}-1\right)^{2},
\end{gathered}
$$

where

$$
Q=\left(\begin{array}{cccc}
-2.3 & 1 & 0.5 & 0 \\
1 & -0.75 & 0 & 0.5 \\
0.5 & 0 & -1 & 0 \\
0 & 0.5 & 0 & -1
\end{array}\right)
$$

is a negative definite matrix. Hence, we can take

$$
\begin{aligned}
\psi(x) & =-\frac{1}{4} x_{2}^{2}+2.3 x_{1}^{2}\left(x_{1}^{2}-1\right)^{2} \\
& \geq a(x, v)-\chi(|v|) .
\end{aligned}
$$


Next, let

$$
|x|_{\mathcal{W}}=\sqrt{x_{2}^{2}+x_{1}^{2}\left(x_{1}^{2}-1\right)^{2}}
$$

and $\alpha_{3}(s)=\frac{1}{4} s^{2}$, then we obtain

$$
\Psi(x)=\psi(x)+\frac{1}{2} \alpha_{3}\left(|x|_{\mathcal{W}}\right),
$$

and it is easy to check that

$$
b(x)=0 \Rightarrow \Psi(x) \leq-\frac{1}{2} \alpha_{3}\left(|x|_{\mathcal{W}}\right) .
$$

Consequently, $V$ is an ISS CLF for the original system and for any $\varepsilon>0$ there is a $\delta>0$ such that whenever $0<$ $|x|_{\mathcal{W}}<\delta$ there exists some control $|u|<\varepsilon$ such that

$$
\Psi(x)+b(x) u<0,
$$

then SCP holds, and the control (13) should be continuous in $\mathbb{R}^{2}$.

The behavior of the controlled system is shown in Fig. 1. Note that with the applied control the two unstable foci become almost attractive in the closed-loop system. Observe that by starting the system from the same initial conditions the control action forces the trajectories to different attractors. To generate the plots the noise signals were chosen $v_{1}(t)=$ $v_{2}(t)=0.2 \sin (10 t)$.

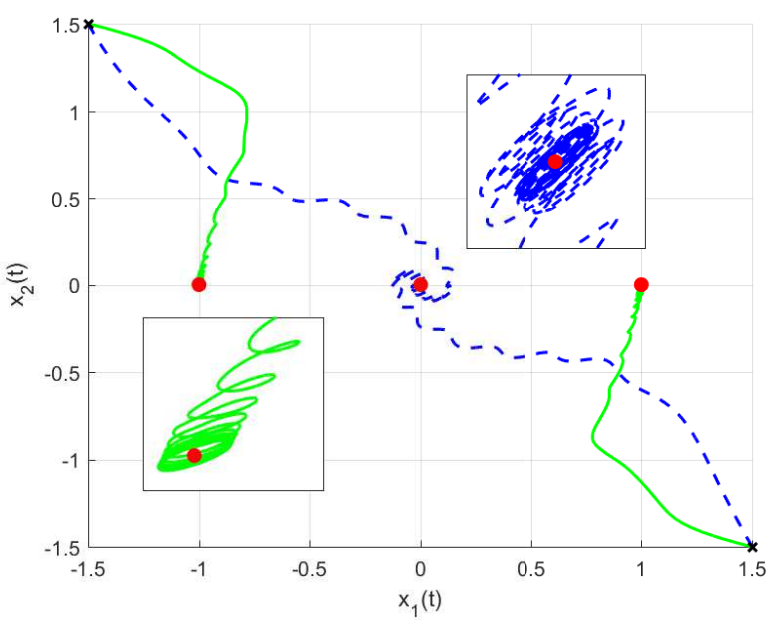

Figure 1: A trajectory convergence for the system with disturbance: without control (dashed line), with control (solid line)

\section{B. Brockett oscillator}

This system has the following model:

$$
\begin{aligned}
\dot{x}_{1}(t)= & x_{2}(t)+v_{1}(t), \\
\dot{x}_{2}(t)= & -x_{1}(t)-\beta x_{2}(t)\left(|x(t)|^{2}-1\right) \\
& +\alpha u(t)+v_{2}(t),
\end{aligned}
$$

where again $x(t)=\left(x_{1}(t) x_{2}(t)\right)^{\top} \in \mathcal{M}=\mathbb{R}^{2}, u(t) \in \mathbb{R}$ and $v(t)=\left(v_{1}(t) v_{2}(t)\right)^{\top} \in \mathbb{R}^{2} ; \alpha>0$ and $\beta>0$ are constant parameters. For $u=0$ and $v=0$ the system has the equilibrium at the origin and an attracting from almost all initial conditions limit cycle on the unit sphere $\mathbb{S}=\{x \in$ $\left.\mathbb{R}^{2}:|x|=1\right\}$, thus, $\mathcal{W}=\{(0,0), \mathbb{S}\}[1]$.

Assume that $\mathcal{A}=\{(0,0)\}$, which in our example will be locally attracting, and choose in this case as a CLF:

$$
V(x)=\frac{1}{2}\left(x_{1}+x_{2}\right)^{2}+\frac{1}{2} x_{1}^{2},
$$

whose derivative for the Brockett oscillator admits

$$
\begin{gathered}
a(x, v)=-x_{1}^{2}+\left(x_{1}+x_{2}\right) x_{2}\left(1+\beta-\beta|x|^{2}\right) \\
+\left(2 x_{1}+x_{2}\right) v_{1}+\left(x_{1}+x_{2}\right) v_{2}, \\
b(x)=\alpha\left(x_{1}+x_{2}\right) .
\end{gathered}
$$

Select $\chi(s)=s^{2}$, and similarly we get

$$
\begin{gathered}
a(x, v)-\chi(|v|)=\left(\begin{array}{c}
x_{1} \\
x_{1}+x_{2} \\
v_{1} \\
v_{2}
\end{array}\right)^{\top} Q\left(\begin{array}{c}
x_{1} \\
x_{1}+x_{2} \\
v_{1} \\
v_{2}
\end{array}\right) \\
-\frac{1}{2} x_{1}^{2}+\left(x_{1}+x_{2}\right)^{2}+\left(x_{1}+x_{2}\right) x_{2}\left[1+\beta-\beta|x|^{2}\right],
\end{gathered}
$$

where

$$
Q=\left(\begin{array}{cccc}
-0.5 & 0 & 0.5 & 0 \\
0 & -1 & 0.5 & 0.5 \\
0.5 & 0.5 & -1 & 0 \\
0 & 0.5 & 0 & -1
\end{array}\right)
$$

is a negative definite matrix. Therefore, we can accept $\psi(x)=-\frac{1}{2} x_{1}^{2}+\left(x_{1}+x_{2}\right)^{2}+\left(x_{1}+x_{2}\right) x_{2}\left(1+\beta-\beta|x|^{2}\right)$, and, finally take

$$
|x|_{\mathcal{W}}=\frac{|x|}{\sqrt{1+|x|^{2}}} \sqrt{\left.|1-| x\right|^{2} \mid}
$$

with $\alpha_{3}(s)=\frac{1}{4} s^{2}$. Then, as before

$$
\Psi(x)=\psi(x)+\frac{1}{2} \alpha_{3}\left(|x|_{\mathcal{W}}\right),
$$

and the main restriction for $V$ to be a CLF, i.e.

$$
b(x)=0 \Rightarrow \Psi(x) \leq-\frac{1}{2} \alpha_{3}\left(|x|_{\mathcal{W}}\right)
$$

is satisfied and in fact, $V$ is an ISS CLF for the original system. However, it is impossible to demonstrate the SCP property in this case due to the term $\left(x_{1}+x_{2}\right) x_{2}\left(1+\beta-\beta|x|^{2}\right)$ in $\Psi(x)$ which is not approaching to zero while $x$ becomes close to $\mathbb{S}$. Therefore, in this example the control (13) will be discontinuous on $\mathbb{S}$.

The effect of the noise for both the open-loop and closedloop systems with $\alpha=\beta=1$ is shown in Fig. 2 Again, observe that by initiating the system from the same initial conditions the control action forces the trajectories to different attractors. In simulation the noise signals also were chosen as $v_{1}(t)=v_{2}(t)=0.2 \sin (10 t)$. 


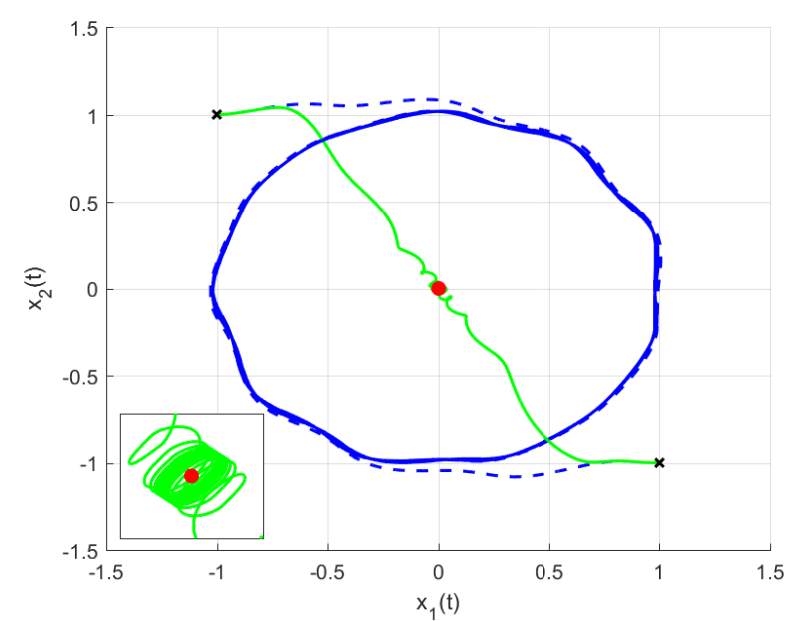

Figure 2: A trajectory convergence for the system with disturbance: without control (dashed line), with control (solid line)

\section{CONCLUSION}

In this work we dealt with a subclass of nonlinear multistable dynamical systems affine in the input. The main problem addressed here was to establish conditions for the existence of a feedback control that renders the ISS (iISS) stability property with respect to disturbances to the closedloop system. In this direction, we have properly extended the notions of ISS (iISS) control Lyapunov functions and small control property within the multistability framework. In the multistable sense, the existence of a ISS (iISS) CLF satisfying the SCP implies the existence of a feedback control law that can be explicitly designed by the universal formula strategy. To exemplify the ISS (iISS) CLF application such a feedback control was designed to robustly stabilize two different nonlinear multistable systems.

\section{REFERENCES}

[1] H. Ahmed, R. Ushirobira, and D. Efimov. Robust global synchronization of Brockett oscillators. IEEE Transactions on Control of Network Systems, 2018. 5

[2] D. Angeli. An almost global notion of input-to-state stability. IEEE Trans. Automatic Control, 49:866-874, 2004. 1

[3] D. Angeli and D. Efimov. Characterization of input-to-state stability for systems with multiple invariant sets. IEEE Transactions on Automatic Control, 60:3242-3256, 2015. 1] 2] 3

[4] D. Angeli, J. E. Ferrell, and E. D. Sontag. Detection of multistability, bifurcations and hysteresis in a large class of biological positivefeedback systems. Proc. Natl. Acad. Sci. USA, 101:1822-1827, 2004. 1.

[5] D. Angeli, E. D. Sontag, and Y. Wang. A characterization of integral input-to-state stability. IEEE Transactions on Automatic Control, 45:1082-1097, 2000. 1 2

[6] Z. Artstein. Stabilization with relaxed controls. Nonlinear Analysis, Theory, Methods and Applications, 7:1163-1173, 1983. 1.4

[7] M. Chaves, T. Eissing, and F. Allgöwer. Bistable biological systems: A characterization through local compact input-to-state stability. IEEE Trans. Automatic Control, 45:87-100, 2008. 1

[8] S. N. Dashkovskiy, D. Efimov, and E. D. Sontag. Input to state stability and allied system properties. Automation and Remote Control, 72:1579-1614, 2011. 1] 2
[9] D. Dudkowski, S. Jafari, T. Kapitaniak, N. V. Kuznetsov, G. A. Leonov, and A. Prasad. Hidden attractors in dynamical systems. Physics Reports, 637:1-50, 2016. 1]

[10] D. Efimov. A condition of clf existence for affine systems. Proceedings of the 41st IEEE Conference on Decision and Control, pages 18821887, 2002. 1

[11] D. Efimov. Universal formula for output asymptotic stabilization. IFAC Proceedings Volumes, 35:239-244, 2002. 1

[12] D. Efimov. Global lyapunov analysis of multistable nonlinear systems. SIAM Journal on Control and Optimization, 50(5):3132-3154, 2012. 1

[13] D. Efimov, J. Schiffer, N. Barabanov, and R. Ortega. A relaxed characterization of iss for periodic systems with multiple invariant sets. European Journal of Control, 37:1-7, 2017. 1.

[14] G. Enciso and E.D. Sontag. Monotone systems under positive feedback: multistability and a reduction theorem. Systems \& Control Lett., 54:159-168, 2005. 1

[15] P. Forni and D. Angeli. The iss approach to the stability and robustness properties of nonautonomous systems with decomposable invariant sets: An overview. European Journal of Control, 30:50-60, 2016. 1

[16] P. Forni and D. Angeli. Characterization of integral input-to-state stability with multiple invariant sets. IEEE Transactions on Automatic Control, 62:3729-3743, 2017. 1]2 3

[17] P. Forni and R. Sepulchre. Differential analysis of nonlinear systems: Revisiting the pendulum example. In Proc. 53rd IEEE Conference on Decision and Control, pages 3848-3859, Los Angeles, US, 2014. 1

[18] J. Guckenheimer and P.J. Holmes. Nonlinear Oscillations, Dynamical Systems, and Bifurcations of Vector Fields, volume 42 of Applied Mathematical Sciences. Springer-Verlag, New York, 1983. 1

[19] C. Hayachi. Nonlinear oscillations in physical systems. McGraw-Hill Book Company, New York, 1964. 1

[20] M. Laurent and N. Kellershohn. Multistability: a major means of differentiation and evolution in biological systems. Trends Biochem. Sci., 24:418-422, 1999. 1

[21] D. Liberzon, E. D. Sontag, and Y. Wang. On integral-input-tostate stabilization. Proceedings of the American Control Conference, 3:1598-1602, 1999. 1, 2, 3, 4

[22] D. Liberzon, E. D. Sontag, and Y. Wang. Universal contruction of feedback laws achieving iss and integral-iss disturbance attenuation. System \& Control Letters, 46:111-127, 2002. 1

[23] Z. Nitecki and M. Shub. Filtration, decompositions, explosions. American Journal of Mathematics, 97:1029-1047, 1975. 2

[24] I. Pchelkina and A. L. Fradkov. Control of oscillatory behavior of multispecies populations. Ecological Modelling, 227:1-6, 2012. 1

[25] A. N. Pisarchik and U. Feudel. Control of multistability. Physics Reports, 540:167-218, 2014. 1

[26] N. Rouche, P. Habets, and M. Laloy. Stability Theory by Liapunov's Direct Method. Springer-Verlag, Heidelberg, 1977. 1

[27] V. V. Rumyantsev and A.S. Oziraner. Stability and stabilization of motion with respect to part of variables. Nauka, Moscow, 1987. [in Russian]. 1

[28] E. D. Sontag. A "universal" construction of artstein's theorem on nonlinear stabilization. Systems \& Control Letters, 13:117-123, 1989. 113

[29] E. D. Sontag. Coments on integral variants of iss. Systems \& Control Letters, 34:93-100, 1998. 1

[30] E. D. Sontag and Y. Wang. On characterizations of the input-to-state stability property. Systems \& Control Letters, 24:351-359, 1995. 1] 2

[31] E. D. Sontag and Y. Wang. New characterizations of the input-to-state stability. IEEE Transactions on Automatic Control, 41:1283-1294, 1996. 112

[32] G.-B. Stan and R. Sepulchre. Analysis of interconnected oscillators by dissipativity theory. IEEE Trans. Automatic Control, 52:256-270, 2007. 1

[33] V. I. Vorotnikov. Partial Stability and Control. Birkhauser, Boston, 1998. 1

[34] V. A. Yakubovich, G. A. Leonov, and A. Kh. Gelig. Stability of Stationary Sets in Control Systems with Discontinuous Nonlinearities. World Scientific, Singapore, 2004. [in Russian]. 1] 